\title{
Low-FODMAP Diet and Its Role in Irritable Bowel Syndrome (IBS) Management
}

\section{Abstract}

Introduction: Irritable Bowel Syndrome (IBS) is a functional and multifactorial gastrointestinal disorder characterized by pain, abdominal distention and motility changes, currently diagnosed based on the Rome IV criteria. The efficacy of classic pharmacological, psychological and dietary treatments for this condition are generally low. The Fermentable Oligosaccharides, Disaccharides, Monosaccharides and Polyols (FODMAP) are short chain carbohydrates poorly absorbed at the intestinal level, fermentable by the microbiota and presumably involved in IBS-associated symptomatology.

Aims: To evaluate the efficacy, feasibility and acceptability of a FODMAP-restricted feeding approach in the relief of symptomatology and in the improvement of the quality of life of patients with IBS, determining the reintroduction of the FODMAP food subgroup (s) involved in the symptom exacerbation.

Materials and Methods: After assessing the existence of initial emotional disorders through the Hospital Anxiety and Depression Scale (HADS) and eating habits, through dietary history, patients diagnosed with IBS were put on a FODMAP-restricted diet for 6 weeks. During this period, the weekly evolution of symptom frequency was assessed. At the end, tests were undertaken to discover the global evolution of the symptoms through the Irritable Bowel Syndrome - Global Assessment Scale (IBS-GAI), the severity of symptomatology through the Irritable Bowel Syndrome - Severity Scoring System (IBS-SSS) and quality of life through Irritable Bowel Syndrome Quality of Life (IBS-QoL).Subsequently, participants tested their tolerance to various FODMAP subtypes with weekly and isolated reintroduction of these in their diet.

Results: In the 36 participants, with a mean age of 38.8 years, there was a reduction in the total consumption of FODMAP from $22.1 \mathrm{~g}$ to $2.1 \mathrm{~g}(\mathrm{p}<0.001)$. A moderate or substantial improvement in the IBS-GAI was observed in $88.9 \%$. An average reduction of 235 points in the IBS-SSS $(p<0.001)$ and a mean increase of 28.7 in the IBS-QoL $(p<0.001)$ was achieved. The initial anxiety and depression levels were not associated with IBS-SSS and presented an inverse association with the IBS-QoL. There was significant improvement in all symptomatology during the $1^{\text {st }}$ week of total FODMAP restriction, except for constipation with an amelioration observed at the $6^{\text {st }}$ week. There was a frequency of intolerance ranging from $30.8 \%$ for fructans to $80.8 \%$ for lactose with the reintroduction of the FODMAP subtypes.

Conclusion: A FODMAP-restricted diet, implemented over a period of 6 weeks, is effective in reducing the severity and frequency of GI symptoms and improving the quality of life of portuguese patients with IBS.

\section{Conflict of Interest}

There is no conflict of interest 